\title{
Design Tradeoffs for Fiber Composite Fuselages under Dynamic Loads using Structural Optimization
}

\author{
Haroon A. Baluch ${ }^{*}$, M. J. L. van Tooren ${ }^{\dagger}$, and E. J. Schut ${ }^{*}$ \\ Delft University of Technology, Kluyverweg 1, 2629 HS Delft, The Netherlands
}

\begin{abstract}
In this paper a fuselage shell, made of fibers composite material, of a passenger aircraft is optimized to get a minimum weight. Two different structural configurations of foam-filled sandwich and stiffened shells are analyzed under the dynamic loads due to the gust. A knowledge based engineering (KBE) approach is formulized to automate the multidisciplinary optimization problem in three different layers. The first layer takes care of the optimization of full fuselage under the constraints of real and negative eigenvalues to get an asymptotically stable closed-loop system. The second layer and third layers, take care of the optimization of the several sections along the length of the fuselage and the optimization of a particular panel of a section, respectively. The constraints in the second and third layers include the critical buckling and wrinkling stresses of the panels and the sections, under bending and torsion loads. The design variables are the stacking sequence of fibers orientations, sizes and positions of the stiffeners in a panel, section length along the longitudinal axis of the fuselage, and the skin thicknesses of the panels. Finally both of the optimized designs are compared with each other in terms of minimum weight, stability and moreover in terms of manufacturing.
\end{abstract}

\section{Introduction}

The use of fiber composite material is getting common in the aircraft industry. Previously their use is quite common only in the light weight aircrafts and in the true sense most of the time the home builders or hobbyists were the forerunners in using these types of materials. The main reason behind this is the ease of manufacturing and its lower cost. We also see that in that period the manufacturers of large airplanes are always reluctant to use the fiber composite material and restricted most of the time to the parts that were less critical to loads. Contrary to that we see a change in late 80 s and early 90 s that few of these manufacturers start using the fiber composite materials in tail-section which was quite encouraging to the world of aircraft engineering and especially to the fiber composite industry. The start of $21^{\text {st }}$ century gives another break through in the sense that, apart from tail section, two of the major manufacturers come up with fiber composite fuselages i.e. the fuselages of A380 and B787. The manufacturers of executive jets have already started producing the complete fiber composites airframes and we expect that those days are not far away when the wings of large airplanes will also be made of fiber composites.

The use of fiber composites has several advantages over the metals which include the better service life in terms of fatigue and moreover the recycling if made of a special kind of fiber composites known as thermoplastics. The knowledge of designing a metal airframe is almost one hundred years old. This knowledge is transferred to us from generations to generations which include the tons of record in the form of experiments and theories with the cases of successes and failures. Presently an aircraft structural designer must be better equipped to deal with the structural analysis of a metal airframe than to a fiber composite one. One of the examples is to find the flexural or torsional stiffness of a fuselage or wing section. The stiffness plays an important role in achieving the stability of the load bearing components of the aircraft, especially when these are exposed to the external disturbances during the atmospheric turbulences and takeoff/landing. In the case of stiffness of a structure made of metal alloys, one has to find the cross-sectional areas and mechanical properties of the material, which for the Aluminum alloys are well known and recorded. In case of fiber composites one has to analytically find the stiffness for each and every section/panel, which on the other hand changes if one of the fiber layers is oriented differently to that of the adjacent section/panel. The lack of vast experience among the aircraft structure design engineers in dealing with the effects of fibers orientations on the stiffness of a structure can be the one of the several reasons behind the slow pace in adapting these materials in the designs of

\footnotetext{
*PhD Researcher, Faculty of Aerospace Engineering, h.a.baluch@tudelft.nl, AIAA Student Member

† Professor Dr., Faculty of Aerospace Engineering, m.j.1.vanTooren@tudelft.nl, AIAA MDO TC Member

${ }^{\ddagger}$ PhD Researcher, Faculty of Aerospace Engineering, e.j.schut@tudelft.nl, AIAA Member
} 
commercial aircrafts, which on the other hand makes sense when the safety of the passengers is the major criteria in certifying a design.

Here it is not attempted to study the effects of the fibers orientations on the stiffness and the consequent strength of an aircraft component in particular but to formulate an optimization algorithm that deals with several design variables to optimize the aft fuselage structure in the concept/preliminary design phase. The stiffness of the vibrating fuselage greatly affects the stability of the whole aircraft in the form of tail loads. The objective is to minimize the weight against the constraints of section/panel wise bucking strength and moreover the real and negative eigenvalues of a whole fuselage structure, which makes a stable closed loop system under dynamic loads.

Here it is appropriate to discuss the past literature on the fiber composite fuselage design. Watson ${ }^{1}$ describes a program to redesign the forward fuselage structure of $\mathrm{AV}-8 \mathrm{~B}$ aircraft, made of metals into a comparable fiber composite structure. In the end the weight reduction of around $25 \%$ is achieved. Johnson et. al. ${ }^{2}$ studied the utilization of composites in fuselage structure on the B757 passenger aircraft. Six different concepts of panel designs are presented, which are made of either honeycomb-only panels, laminated stiffeners, or the foam-filled stiffeners with skin, and the hybrid designs of either foam-filled stiffeners or laminated stiffeners with the honeycomb panels. In the end the authors concluded that in terms of weight the combination of foam-filled stiffeners and laminated skin is the best but in terms of manufacturing the fuselage made of honeycomb-only panels require minimal tooling and factory hours. Lately, van Tooren ${ }^{3}$ studied the sandwich fuselage design, which includes the derivations of buckling stress, boundary conditions, stress concentrations around the cutouts, the damage tolerance and lastly an example on the design of a sandwich fuselage structure of Extra-400 light aircraft is presented. At the panel's level Collier ${ }^{4}$ derived the stiffness matrices for the laminated stiffened panels. Later on, Tuttle and Zabinsky ${ }^{5}$ presented the methodology of calculating the effective panel stiffness and strength of a crown panel by using software called COSTADE.

The transformation of fuselage structural designs from metals to fiber composite materials seems to be a change only in the material selection whiles the topology of the cross-section in most of the cases seems to be remain the same i.e. design revolves around the stiffened panels ${ }^{1,2,5}$. The use of sandwich panels in the design of a fuselage structure, which in the present time, can only be found in some light aircrafts whereas their use in a slender fuselage of a passenger aircraft is yet to be seen.

In this paper, a study is carried out for the design tradeoffs of a fuselage structure made of the two different types of panels i.e. foam-filled stiffened panels with laminated skin and foam-filled sandwich panels, which are incorporated separately in three different optimization cases. The design variables are the stacking sequence of fibers orientations, the skin thicknesses of the panels, sizes and positions of stiffeners along the width of a panel, the sandwich thickness and the position of the frames along the length of the fuselage. Section 2 introduces the formulation of the optimization algorithm, which is segregated into three layers where each layer holds its particular objective, and set of constraints and design variables. The methodology of structural representations, and load intensities of the panels and the fuselage are discussed in the subsections A and B, respectively. A brief discussion about the dynamic loads and the mathematical model is presented in section 3 , which is followed by

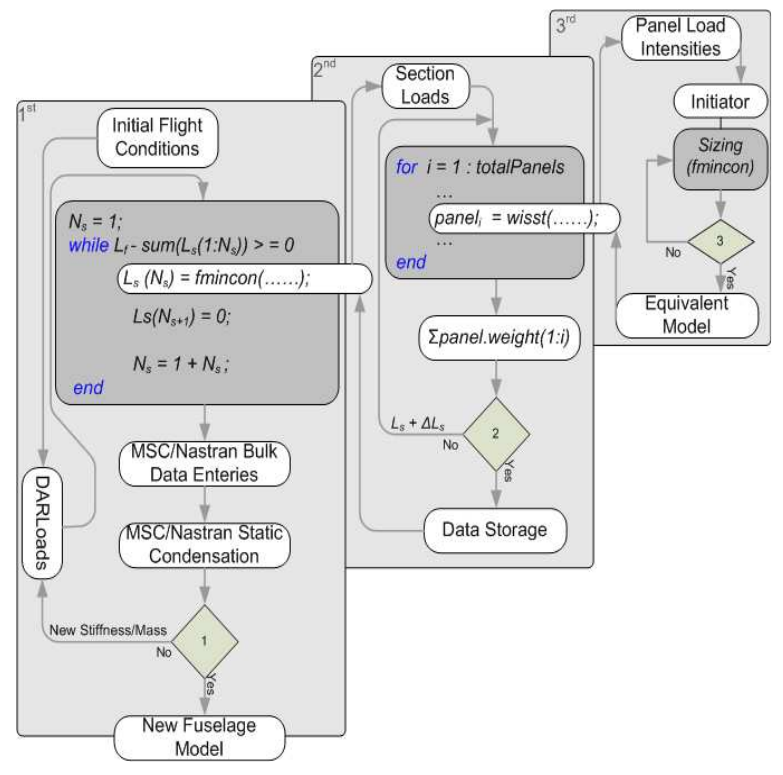

Fig. 1 The optimization algorithm section 4 where an executive aircraft is subjected to dynamic loads due to the gust while, as stated earlier, the aft fuselage is optimized with two different concepts of fibers composite panels. Conclusions with future plans are discussed in section 5 .

\section{The Optimization Algorithm}

The optimization algorithm, which is to be discussed in this section, is formulated under the domain of the "Analysis Tools" section of the Design and Engineering Engine (DEE), which is a knowledge based engineering (KBE) tool to automate the process of multidisciplinary optimization in aircraft $\operatorname{design}^{6,7}$. The algorithm is specifically developed for the structural optimization of a fuselage structure and as for as other components like wings and stabilizers are concerned, a small change in constraint equations and a few design variables can let a design engineer to use the same routine. 


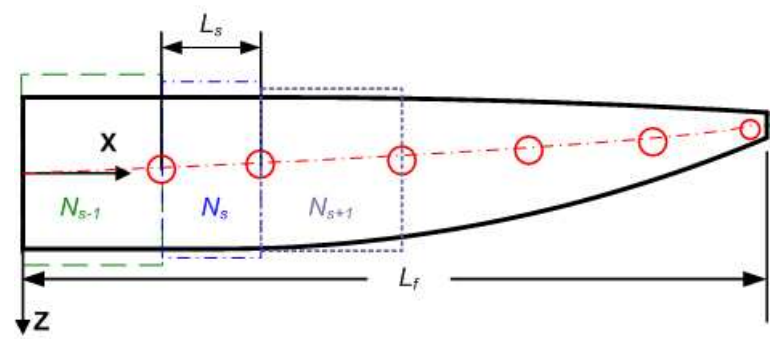

Fig. 2 Aft fuselage

The algorithm is segregated into three layers, see Fig 1. In which the first or the upper most layer starts with the inputs for the initial conditions of the aircraft, which includes the flight conditions, aerodynamics, and the structural properties of the whole aircraft that also includes the initial structure which is to optimized later on. The initial conditions in the form of structured arrays are then transferred to DARLoads for the dynamic loads analysis. DARLoads is a software tool for the dynamic loads analysis for the flexible aircrafts, which is being developed by the DAR group of the Faculty of Aerospace Engineering in Delft University ${ }^{8}$, see section 3. DARLoads gives the output in the form of internal structural loads and deflections of all the components of the aircraft, which include wings, fuselage and empennage.

DARLoads is being followed by a while loop, which optimizes the length $L_{s}$ of a section $N_{s}$ of the aft fuselage, see Fig. 2, where the $L_{s}$ represents the length between the two adjacent fuselage frames. The while loop begins with a section number and whenever the length $L_{s}$ of current section $N_{s}$ is optimized by the use of downstream layers, the $N_{s}$ is incremented to the next section number. It is supposed that the total length $L_{f}$ of the aft fuselage is fixed during the preliminary sizing of the aircraft so the condition to enter in the while loop is decided by the sum of all the optimized lengths of the previous sections should not exceed the total length of the aft fuselage, which can also acts a design space for the next section i.e. the upper bound of $L_{s}$, see section 4 .

Table 1 Optimization variables, constraints and objective

Local Panel Level

Local Section Level

Global Structural Level

Objective

$$
\min _{\bar{x}} f(\bar{x})
$$

$$
f(x)=\frac{\text { Weight }_{\text {panel }}}{L_{s}}
$$

$$
\sum_{1}^{\text {totalPanels }} \frac{\text { Weight }_{\text {panels }}}{L_{s}}
$$$$
\sum_{1}^{i=\text { totalSections }} \frac{\text { Weight }_{\text {Sections }_{i}}}{L_{s_{i}}}
$$

$\begin{array}{cl} & \mathrm{h}=\text { panel height } \\ & \mathrm{t}_{1}=\text { facing thickness } \\ & \mathrm{t}_{2}=\text { stiffener thickness } \\ \text { Design } & \mathrm{n}_{1}=\text { facing stacking } \\ \text { variables } & \text { seq. } \\ & \mathrm{n}_{2}=\text { stiffener stacking } \\ & \text { seq. } \\ & \mathrm{w}_{1}=\text { stiffener spacing } \\ & \mathrm{w}_{2}=\text { stiffener width }\end{array}$

Material failure (Tsai-Hill)

Constraints Skin (facing) buckling loads Stiffener wrinkling loads Panel buckling loads
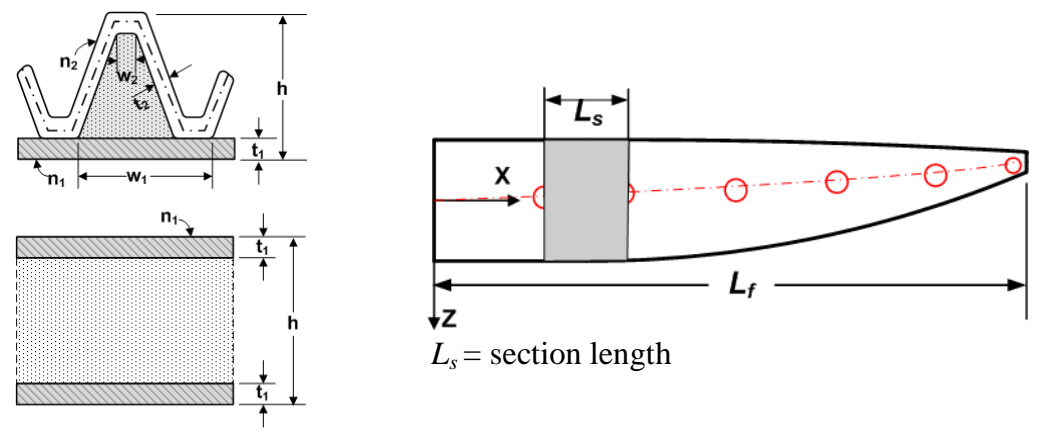

$\operatorname{eig}\left(M_{A F}-\lambda K_{A F}\right) \leq 0$

Torsion buckling loads

eig = eigenvalue solution

$M_{A F}=$ gen. mass matrix

$K_{A F}=$ gen. stiffness matrix

The while loop calls the $2^{\text {nd }}$ layer by using the fmincon optimization function in Matlab ${ }^{9}$. Loads in the form of shear forces in three directions and corresponding bending/torsion moments, at the root of the current section, are read from the DARLoads output. A for loop is called afterwards, where each panel in the current section is optimized through the $3^{\text {rd }}$ layer of the algorithm. The length of a for loop depends upon the number of panels considered in a circular section of the fuselage. For e.g. the length of the for loop is 04, if a fuselage is discretized into four panels i.e. crown on the top, keel at the bottom, and two sides ${ }^{2}$, but in this paper a fuselage cross-section is discretized into 12 straight panels, see subsection A.

For the turn by turn optimization of each panel, the computer program known as $\mathrm{WISST}^{7}$ is called in each loop. Depending upon the position of a panel along the circumference of the fuselage, the loads in the $3^{\text {rd }}$ layer 
are converted into the load intensities of the panel under consideration. Depending upon the panel type i.e. stiffened or sandwiched, WISST first initiates a feasible solution of the certain width out of the total given width of the panel. Readers are referred to Fig. 5 and 6 of Ref. 7, where a stiffened panel is depicted in the form of a stiffener and called as a blade element. The failure criteria taken into account are the strength and stability of the blade element. The solution from the initiator is then transferred to the sizing tool, which takes account of the constraints of ply strength and buckling of a full-width panel and meanwhile minimizing the objective of weight per length of the panel. Whenever the objective is achieved the optimized panel is transformed into an equivalent sandwich panel with a fixed skin thickness for each panel along the circumference of the fuselage but with different equivalent material properties of both skin and core i.e. modulus of elasticity/rigidity, poison ratio, and density etc. These equivalent properties are replaced with the new values, till the objective and constraints with respect to the section length $L_{s}$ are achieved in the $2^{\text {nd }}$ layer of the optimization. Table 1 gives a brief overview of the design variables, constrains, and objectives from local panel to full aft fuselage structural level, where the mathematical expressions related to panel and section constraints are stated in Appendices.

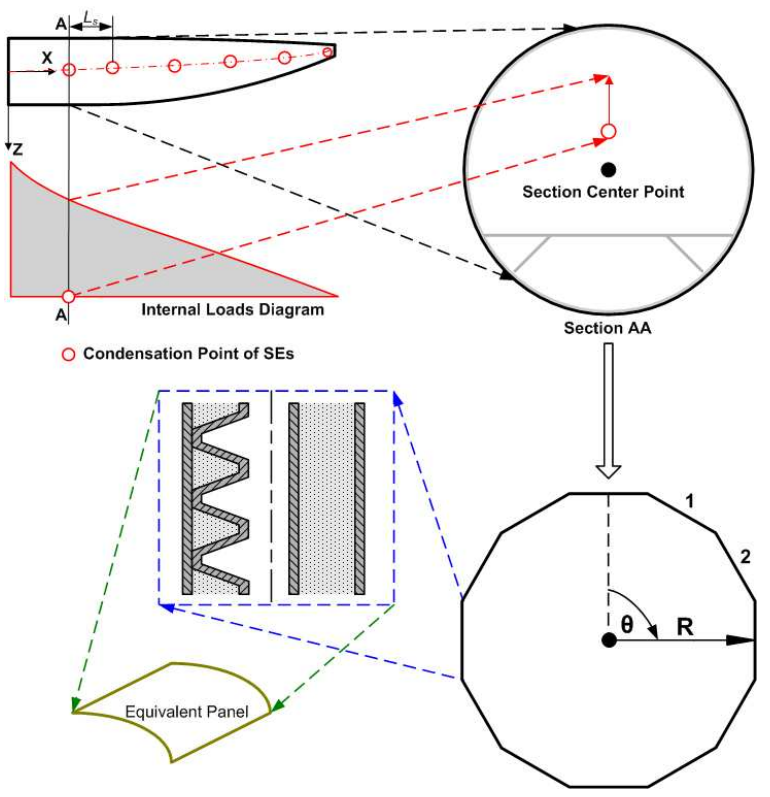

Fig. 3 The structural representation of the fuselage

circumference. Figure 3 shows the geometry of an aft fuselage, where a circular cross-section is transformed into 12 straight panels. For better accuracy, the number of panels along the circumference can be increased, which may also increase the solution time.

The loads are applied at the condensation point, which rests at the root of the current section. The loads are interpolated from the given shear and bending/torsion moment diagrams. The condensation point represents the current neutral axis of the section and it may change its position in both vertical and horizontal directions that depend upon the weight of the each panel optimized in the $2^{\text {nd }}$ and $3^{\text {rd }}$ layers of the optimization algorithm. Depending upon the concept used in the fuselage structural design, each panel is shown to be represented in the form of foam-filled stiffened panel or foam-filled sandwich panel.

As stated above an optimized panel is further transformed into an equivalent sandwich panel, which is to be used in the static condensation by using the MSC/Nastran ${ }^{11}$. Each section is model as a superelement (SE) by using the SESET bulk data entry, where the grids at the root of that section represent its interior points and the grids at the tip acts as its
When the upper bound of the design space, as given in the condition to enter in while loop, is exhausted, the equivalent model for each panel is written out in a text file, which includes the bulk data entries of grids and corresponding quad elements with particular equivalent material properties. MSC/Nastran is called on for the static condensation which gives the new stiffness and mass matrices of the aft fuselage, which are then assembled with the rest of the components in global stiffness and mass matrices of the whole aircraft. If the constraint of real and negative eigenvalues of the aft fuselage structure is not achieved then the DARLoads is called on again with the new stiffness and mass matrices, which in turn gives the new sets of loads for the next iteration.

\section{A. Structural Representation}

The circular section of a fuselage is presented in the form of straight and rectangular panels, placed at the particular radius of a section with an angle $\theta$ along the

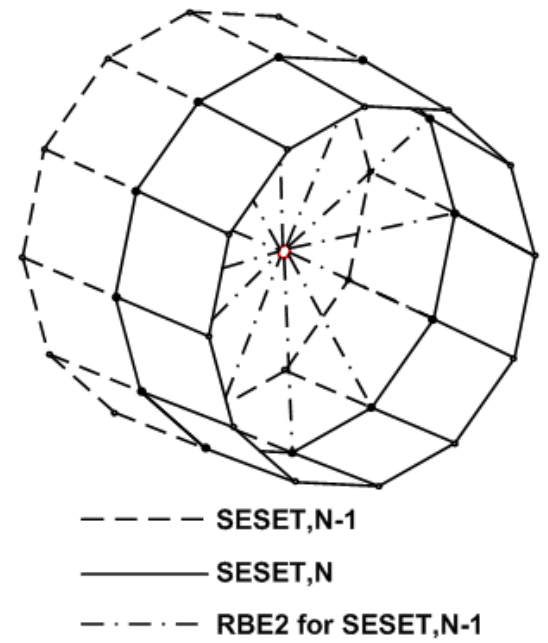

Exterior Grids for SESET,N-1

Condensation Grid

Fig. 4 The superelement representation 


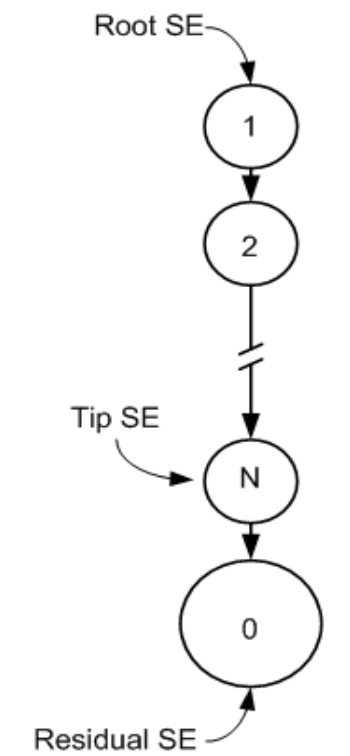

Fig. 5 Superelement tree

exterior points to the next section, see Fig. 4. Figure 5 shows the superelement tree from root to the tip superelement. By using the rigid body element, RBE2, the stiffness and mass properties of the section are condensed to the grid at neutral axis. The output data from the MSC/Nastran is written out in output4 format, which is then read by the Matlab to get the new mass and stiffness matrices of the aft fuselage structure.

\section{B. Panel Load Intensities}

The forces in shear are combined and the magnitude of the force perpendicular to the point is taken as the equivalent shear force in the panel ${ }^{12}$. The shear flow is positive in clock wise direction to the reference shown in Fig 3.

$$
\begin{aligned}
& p_{x y_{1}}=\frac{F_{z} \sin \theta-F_{y} \cos \theta}{\pi R} \\
& p_{x y_{2}}=\frac{M_{x}}{\pi R^{2}} \\
& p_{x y}=\operatorname{abs}\left(p_{x y_{1}}+p_{x y_{2}}\right)
\end{aligned}
$$

where $F_{z}, F_{y}$ and $M_{x}$ are the shear forces in $y$ and $z$-axis, and torsion moment along the $x$-axis, respectively. The loads intensities in compression arise due the bending moments $M_{y}$ and $M_{z}$, axial shear force $F_{x}$, and the internal pressure $p$, which is taken as around $0.1 \mathrm{MPa}^{12}$ :

$$
\begin{aligned}
& p_{x_{1}}=\frac{F_{x}}{2 \pi R} \\
& p_{x_{2}}=\frac{M_{z} \sin \theta+M_{y} \cos \theta}{\pi R^{2}} \\
& p_{x_{3}}=\frac{p R}{2} \\
& p_{x}=p_{x_{1}}+p_{x_{2}}+p_{x_{3}}
\end{aligned}
$$

The load intensity along the hoop direction is also caused by the internal pressure:

$$
p_{y}=p R
$$

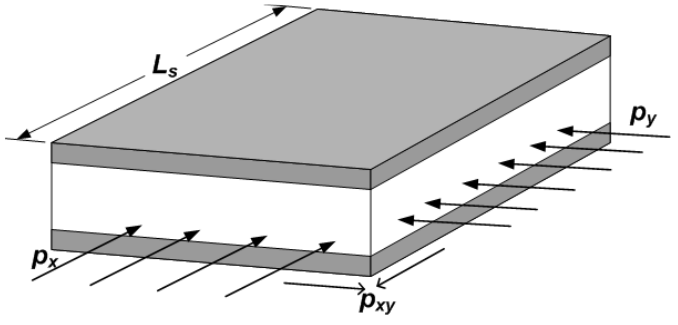

Fig. 6 Panel load intensities representation

\section{An Overview of Dynamic Loads}

Dynamic loads are calculated by solving the inertially coupled equations of motion (EoM) of a fully flexible aircraft ${ }^{13}$. The computer code DARLoads accepts the aircraft structural data in the form of local stiffness and lumped mass elements. The aerodynamics data is given in the form of local strips on each lifting surface, where each strip is defined with its particular quasi-steady lifting coefficients. All the component level stiffness, mass and aerodynamic influence coefficients are assembled in the global matrices of the full aircraft, which are then solved in state space form.

The aircraft motion is distinguished into rigid body motions with respect to inertial axes on ground, and the elastic motions of aircraft structural components with respect to aircraft body axes. Considering the elastic motions or vibrations about equilibrium state are smaller in magnitude to those of rigid motions, EoM can be written into state-space form and linearized into zero and firstorder equations by perturbation theory of extended aeroelasticity ${ }^{13}$ :

American Institute of Aeronautics and Astronautics 


$$
\dot{x}^{(0)}(t)=A^{(0)}\left(x^{(0)}(t)\right)+B^{(0)}\left(x^{(0)}(t)\right) u^{(0)}(t)
$$

The above equation introduces the zero-order state-vector $x^{(0)}$ that represents the rigid body motions i.e. translations and rotations with respect to inertial. The control vector, $u^{(0)}=\left[\begin{array}{lll}\delta_{e}^{(0)} \delta_{a}^{(0)} & \delta_{r}^{(0)} & \delta_{T}^{(0)}\end{array}\right]^{\mathrm{T}}$, represents the zero-order control inputs of elevator, aileron, rudder and thrust, respectively. State space matrices $A^{(0)}$ and $B^{(0)}$ represent the coefficient matrices for inertia and control forces, respectively. During the steady-state flight the zero-order coefficient matrices, $A^{(0)}$ and $B^{(0)}$, remain constant and so is the zero-order state vector $x^{(0)}(t)$. The first-order vector $x^{(1)}(t)$ which takes account of vibrations and their effects on overall response of the aircraft:

$$
\dot{x}^{(1)}(t)=\left(A^{*}+B_{x}-B_{u} G\right) x^{(1)}(t)+F_{\text {ext }}(t)
$$

The state-matrix $A^{*}$ contains the partial derivatives of zero-order velocities, stiffness, and damping matrices with respect to the first-order state-vector. The coefficient matrix $B_{x}$ gives the sum of aerodynamic and gravitational forces and subsequent moments due to vehicle motion resulting from external disturbance $F_{\text {ext }} . B_{u}$ multiplied by the closed-loop gain matrix $G$ gives the coefficients of forces and moments due to first-order control inputs which consequently minimize the effects of external disturbance.

Using the mode displacement method, which is based on the internal elastic forces, the total loads along certain degree of freedom (d.o.f) ' $u$ ' of a component ' $i$ ' are expressed as the sum of static loads and time integration of the dynamic loads over the steady state:

$$
L_{i_{(u)}}=\varphi_{i_{(u)}} K_{i_{(u)}}\left(x_{i_{(u)}}^{(0)}+\int_{0}^{\tau} x_{i_{(u)}}^{(1)}(t) d t\right)
$$

where $K$ and $\varphi$ are the stiffness matrix and vector of mode shapes of the component, respectively.

\section{Optimization Example}

Table 2 Aircraft geometry and flight conditions

\begin{tabular}{ll}
\hline \hline Airspeed, $\mathrm{V}^{(0)}, \mathrm{m}^{-\mathrm{sec}^{-1}}$ & 250 \\
Altitude, $\mathrm{m}$ & 7620.0 \\
Wing Span, m & 16.7 \\
Mass, Kg & 5884.23 \\
Radius at the root of the aft fuselage, $\mathrm{m}$ & 0.7 \\
Radius at the tip of the aft fuselage, $\mathrm{m}$ & 0.06 \\
Total Length of the fuselage $L_{f}, \mathrm{~m}$ & 7.1 \\
Max. panel width, $\mathrm{m}$ & 0.365 \\
\hline \hline
\end{tabular}

A Twin-jet aircraft is selected as a test case over a discrete gust. To get the initial loads set, the input data required for the structural and aerodynamics properties of the aircraft are taken from the Ref. 14. Flight conditions for symmetric flight with the dimensions of the outer geometry of the aft fuselage are given in Table 2. After reading the inputs DARLoads assembles all the required matrices. To get the trim condition, DARLoads minimizes the quadric function of rigid-body zero order state vector in Eq. (4) and optimizes the control vector for the given speed ${ }^{8}$. The external disturbance in the form of discrete gust is applied for a period of $1 \mathrm{sec}$, and Eq. (5) is numerically solved over a $10 \mathrm{sec}$. Response of the aircraft in the form of both rigid and elastic motions are recorded and loads along each d.o.f are extracted by using the Eq. (6). Figure 7 shows the static loads along the length of the aft fuselage during the trim conditions, where the Fig. 8-10 show the dynamic loads at the root section of the fuselage over the time period of simulation. The sign convention for the loads is to be followed with respect to the axes system shown in Fig 3.

To start with the structural optimization problem, first the optimization for a foam-filled sandwich fuselage structure is initiated. Material used in the analysis is given in the Table 3 . It is thought that a sandwich panels should have a higher buckling strength to that of a stiffened panel so the upper bound of the design variable $L_{s}$ in the $2^{\text {nd }}$ layer of the optimization is taken same as the while loop condition given in the $1^{\text {st }}$ layer of the optimization. The lower bound is given as 0.1 meters. While using the clockwise direction and starting from the panel 1, as shown in Fig. 3, the $2^{\text {nd }}$ layer calls the $3^{\text {rd }}$ layer for the optimization of each panel. Meanwhile the loads sets
Table 3 Material Properties

\begin{tabular}{ll}
\hline \hline \multicolumn{2}{c}{ Carbon fiber fabric } \\
Flexural modulus, $\mathrm{E}_{11}=\mathrm{E}_{22}, \mathrm{~N}-\mathrm{mm}^{-2}$ & 45000.0 \\
Shear modulus, $\mathrm{G}_{12}, \mathrm{~N}-\mathrm{mm}^{-2}$ & 4000.0 \\
Poisson ratio, $\nu_{12}$ & 0.03 \\
Density, Kg- $\mathrm{mm}^{-3} \quad 1.561 \mathrm{e}-6$ \\
\multicolumn{1}{c}{ Foam core } \\
Flexural modulus, $\mathrm{E}_{11}=\mathrm{E}_{22}, \mathrm{~N}-\mathrm{mm}^{-2}$ & 75.0 \\
Shear modulus, $\mathrm{G}_{12}, \mathrm{~N}-\mathrm{mm}^{-2}$ & 24.0 \\
Poisson ratio, $\nu_{12}$ & 0.0 \\
Density, Kg- $\mathrm{mm}^{-3}$ & $52 \mathrm{e}-9$ \\
\hline
\end{tabular}




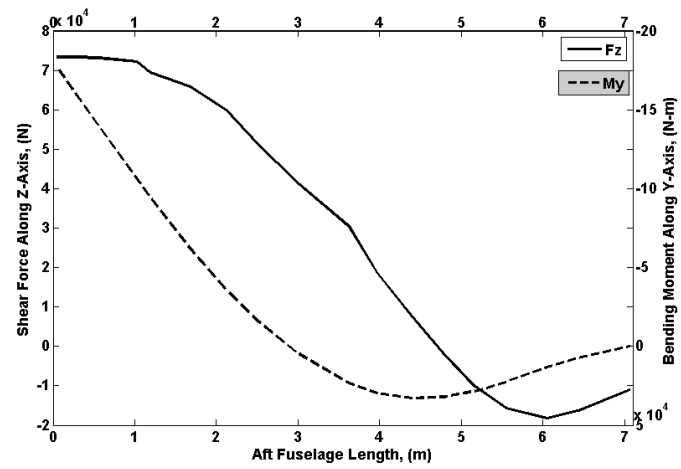

Fig. 7 Static shear and moments in Z-Y Plane

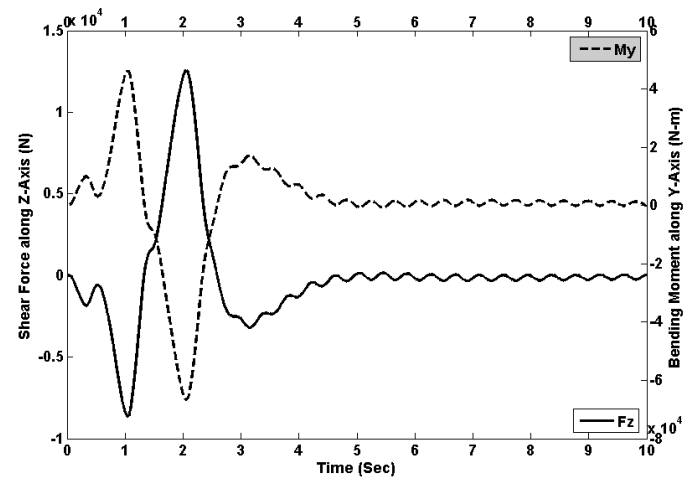

Fig. 9 Dynamic shear and moments in Z-Y Plane

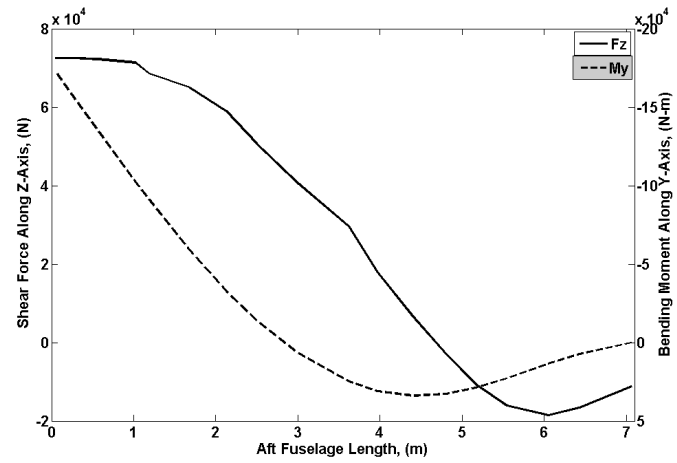

Fig. 11 Total shear and moments in Z-Y Plane

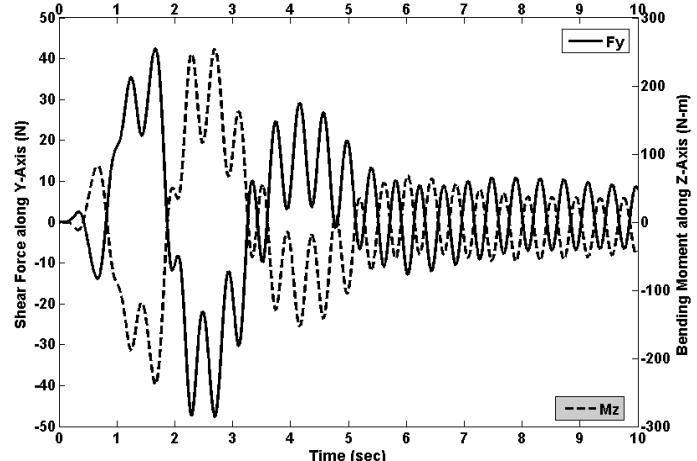

Fig. 8 Dynamic shear and moments in Y-Z plane

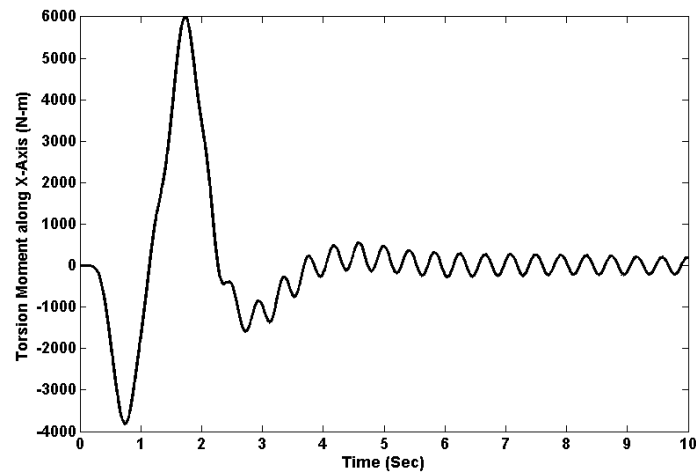

Fig. 10 Dynamic torsion moment along $\mathrm{X}$-axis

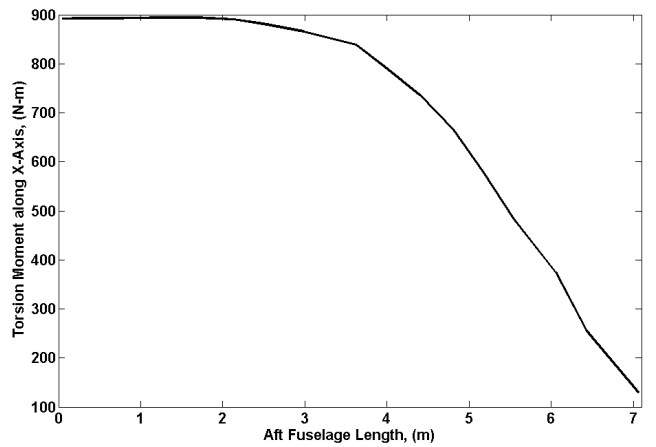

Fig. 12 Total torsion moment along $\mathrm{X}$-axis

that are given in Fig. 7-10 are integrated by using the Eq. (6) and presented in Fig. 11 and 12. By using the Eqs. (1-3) the load vectors are converted into the load intensities for each panel. The length of the first section is optimized at 3.62 meters. Consequently the optimizer suggests only two sections with approximately the same lengths but different weights. The optimized lengths and corresponding weights of the fuselage sections are given in Table 4.

While keeping in mind the trend of the optimized section lengths in a sandwich structure, the foam-filled stiffened panel optimization is initiated in the second case with a fixed upper bound i.e. a length of 1.2 meters. The results are pretty much different to those of the first case and the fuselage is optimized into six sections with diverging weights. Table 4 shows the section lengths with their weights per lengths. The section length for each of the first five sections is always settled at the upper bound. It shows that the analysis could have proceeded further and optimized the section with the larger lengths but the upper bound always limited them to the given length. The length of the last section is automatically selected as the remaining portion left out of the sum of the optimized section sizes minus the total length $L_{f}$ of the fuselage.

In the next attempt i.e. in Case 3 the upper bound is set at 2.0 meters. The optimized section lengths with their weights per length ratio are also given in Table 4 . The section lengths are fairly improved as compared to those of calculated in case 2 . However, likewise in the previous case, the length of the $4^{\text {th }}$ section also settles at 
Table 4 Section Lengths and Weight Comparison

\begin{tabular}{|c|c|c|c|c|c|c|}
\hline \multirow[b]{2}{*}{$\begin{array}{l}\text { Sec. \# } \\
N_{s}\end{array}$} & \multicolumn{2}{|c|}{$\begin{array}{l}\text { Foam-Filled Sandwich Structure } \\
\text { (Case 1) }\end{array}$} & \multicolumn{2}{|c|}{$\begin{array}{l}\text { Foam-Filled Stiffened Structure } \\
\text { (Case 2) }\end{array}$} & \multicolumn{2}{|c|}{$\begin{array}{l}\text { Foam-Filled Stiffened Structure } \\
\text { (Case 3) }\end{array}$} \\
\hline & $\begin{array}{c}\text { Section } \\
\text { Length } L_{f}, \mathrm{~m}\end{array}$ & $\begin{array}{l}\text { Weight Ratio } \\
\mathrm{Kg}^{-\mathrm{m}^{-1}}\end{array}$ & $\begin{array}{c}\text { Section } \\
\text { Length } L_{f}, \mathrm{~m}\end{array}$ & $\begin{array}{c}\text { Weight Ratio } \\
\text { Kg-m }^{-1}\end{array}$ & $\begin{array}{c}\text { Section } \\
\text { Length } L_{f}, \mathrm{~m}\end{array}$ & $\begin{array}{c}\text { Weight Ratio } \\
\text { Kg-m }^{-1}\end{array}$ \\
\hline 1 & 3.6282 & 238.27 & 1.20 & 59.122 & 1.2237 & 115.13 \\
\hline 2 & 3.4783 & 215.6 & 1.20 & 72.183 & 1.6119 & 66.46 \\
\hline 3 & & & 1.20 & 57.60 & 1.8059 & 62.79 \\
\hline 4 & & & 1.20 & 60.0 & 2.000 & 62.56 \\
\hline 5 & & & 1.200 & 49.20 & 0.465 & 32.31 \\
\hline 6 & & & 1.1065 & 38.00 & & \\
\hline Total W & & 453.87 & & 336.10 & & 339.25 \\
\hline
\end{tabular}

the given upper bound. Figures 13 and 14 plot the panel load intensities from root to tip, which show that the normal load intensity along $x$-axis is mainly changing its direction from top to lower panels i.e. the top panels at the fuselage root are under compression and lower panels are under extension. At the middle of the aft fuselage the normal load intensity along $x$-axis is same all along the circumference. On the other hand, after the middle of the aft fuselage, the top panels are under extension and lower panels are under compression. The reason is the change in the load direction at these sections, which is caused by the load contributed by the empennage, see Fig. 7. The normal load intensity due the internal pressure, which is taken constant all over the length of the fuselage, applies extension loads on the panel. So when the bending moment $M_{y}$ changes its direction, the load intensity due to internal pressure is added to that of the $M_{y}$ in Eq. (2). Whereas due to the small value of bending moment $M_{z}$, it has the minimum effect on the panels loads intensities.

Figure 14-15 show that in the first two sections the side panels with high shear loads are heavier in weights as compared to those of the upper and lower panels. The reason can be the active wrinkling constraints due to shear loads on a stiffener, which let the optimizer to optimize a stiffener with the larger values of stiffener thickness and height. Figure 16-18 show the optimized sizes of the panels. In the case of $3^{\text {rd }}$ and $4^{\text {th }}$ sections the situation is on the other way around i.e. upper and lower panels is heavier than the side panels. The shear loads in these sections is quite low whereas the normal load in extension is the main factor which decides the sizes of a panel. It is also observed that the upper and lower panels which are under dominant normal loads are optimized with a thicker skin as compared to the side panels which are under the combined loadings of normal and shear load intensities. On the other hand, as stated above, the thicknesses of the stiffeners under combined loadings are larger than those of the top and bottom panels. Interestingly, the optimizer compensates the skin thickness of the stiffener with its height or the other way around, which can be observed in few panels, for e.g. panels marked as 'A' in Fig. 17-18. Similarly, the panel level buckling on these panels is higher than the rest of the panels shown in Fig. 19-20, which shows that to counter the buckling in a panel, the skin thickness of a stiffener impacts more than to that of the height of a stiffener.

Here, it is appropriate to discuss the successes and failures in running the above exercise. As for as the panel sizes are concerned, which are being optimized by the third layer of optimization algorithm, do not seem to be very practical in the sense that the thicknesses are not consistent all along the circumference and also along the length of the fuselage structure. Though the optimization routine is achieving the objective of minimum weights with the constraints of the strengths and the buckling loads, but it is not practically feasible to manufacture a fuselage section with divergent panel sizes. The divergent sizes seem to be resulted from the initiator in the panel optimization, where it randomly initiates the feasible values for the optimization of design variables, and for each of the iteration in the $2^{\text {nd }}$ layer of the optimization algorithm the initiator in the $3^{\text {rd }}$ layer generates a unique feasible solution. To improve the optimization problem in the $3^{\text {rd }}$ layer in future, it is thought to start with a panel with the most critical loads and the optimized solution of that panel should be used as the feasible solution for the rest of the panels of a section. Secondly, instead of discretising the fuselage into several straight panels, it would be much better to use the curved panels in the form of crown, keel and two side panels as given in Ref. 2. Keeping in view the divergent results in first two layers, the $1^{\text {st }}$ layer of the optimization is terminated half way that at least gives the results from the first two layers, which are presented here. Apart from the design tradeoff study, the theme of the research also includes the formulation of an optimization algorithm, which is to be used in the structural optimization of the fiber composite fuselage during the concept and preliminary design phases. Besides having some local level technical issues, as stated above, the overall working of the optimization algorithm seems to be quite feasible and can also be used for the metal structure. 


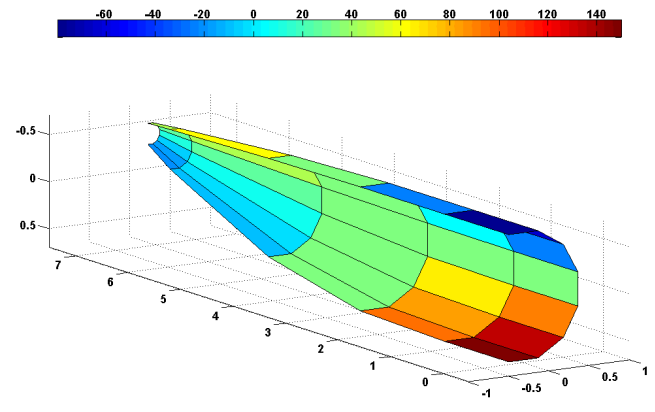

Fig. 13 Normal load intensity along X-axis, N-mm-1

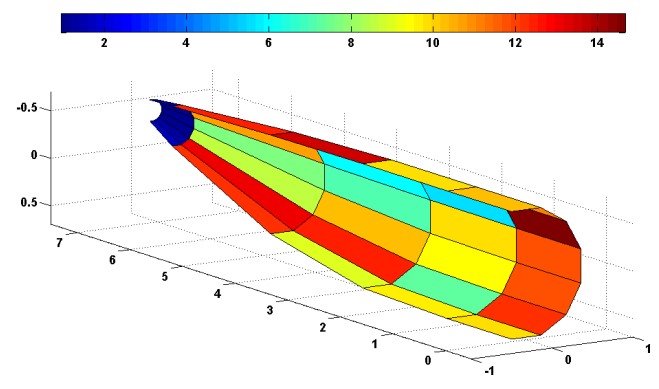

Fig. 15 Panel weight (Case 3), Kg

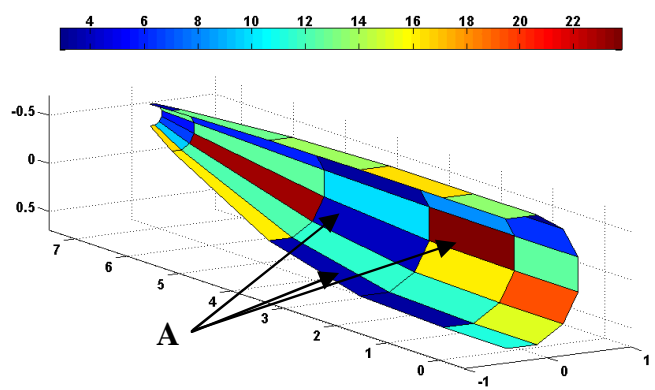

Fig. 17 Stiffener thickness ' $t_{2}$ ' (Case 3), mm

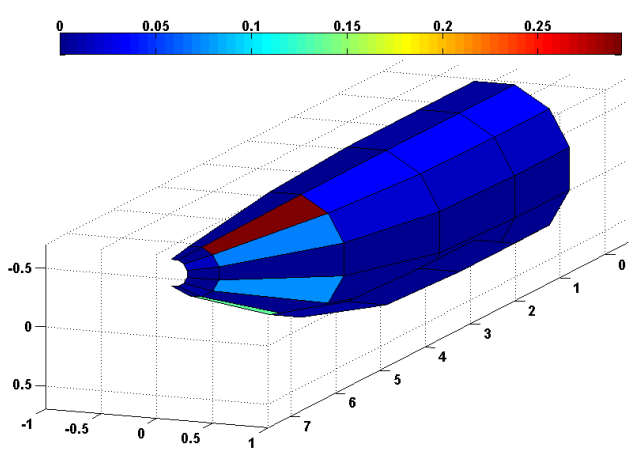

Fig. 19 Stiffener wrinkling state (Case 3)

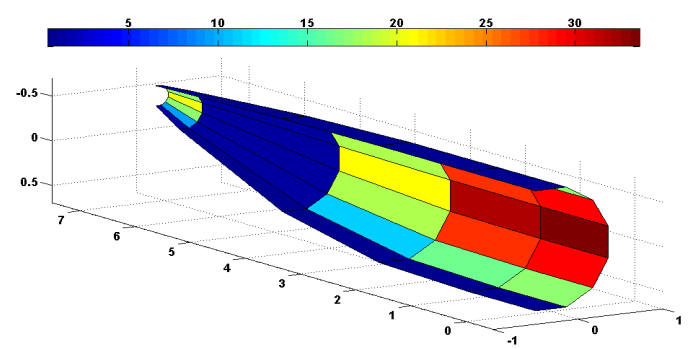

Fig. 14 Shear load intensity in X-Y plane, N-mm-1

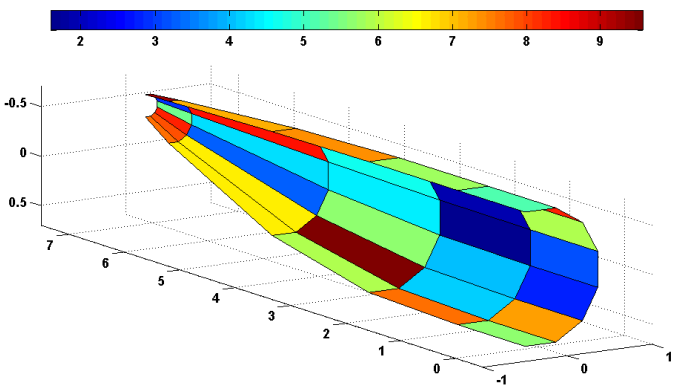

Fig. 16 Panel skin thickness ' $t_{1}$ ' (Case 3), mm

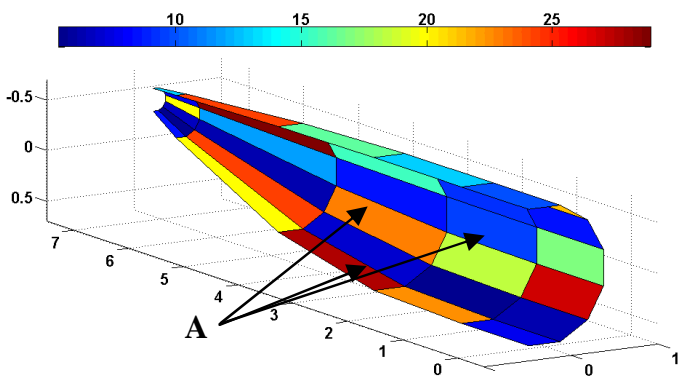

Fig. 18 Panel height 'h' (Case 3), mm

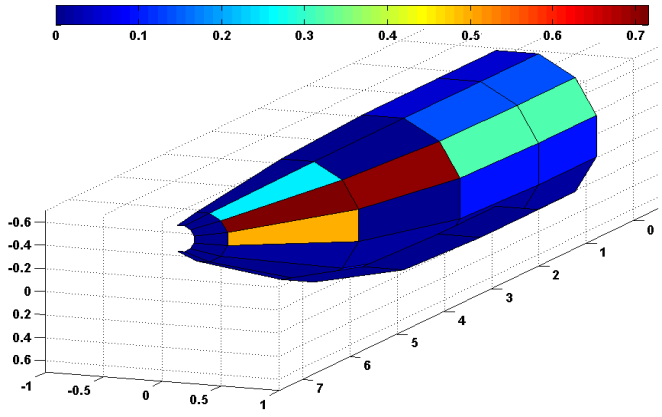

Fig. 20 Panel buckling state (Case 3) 
Now comparing the total weights of both the design concepts i.e. stiffened and sandwich structure. It shows that the foam-filled stiffened panel has an advantage over the sandwich structure. The weight of frames is not included in the design yet, so the weight for the stiffened structure will increase further. However, a practical structural design of the fuselage requires several frames to support the floor and also the connections between the fuselage and wings or tail sections, which make it obvious to include the frames in the sandwich structure too. From this study the only advantage of sandwich structure over the stiffened one appears to be in terms of manufacturing. As stated in Ref. 2, the stiffened panels require several manufacturing processes and factory hours, whereas the sandwich panels are easy to manufacture and require less factory hours.

\section{Conclusions}

An attempt is made to formulize an optimization algorithm, which is to be used for the structural optimization in the fuselage design. The algorithm is divided into three layers of optimization, where each layer has its own objective and constraints functions, and design variables. The first layer optimizes the full fuselage structure, while keeping the constraints of negative and real eigenvalue solution of mass and stiffness matrix. The second and third layers take care of section level and panel level optimization, respectively, where the objective is to minimize the ratio of weight per length of a section or a panel. The constraints in this case are buckling and material strength of the concerned fuselage section or the panel.

The aft fuselage structure of an executive jet is taken as a test case for the optimization. The structure is designed with two types of concepts i.e. foam-filled sandwich panels and foam-filled stiffened panel. Structural loads sets due to a discrete gust input are created and optimization problem for each concept. The results show that the foam-filled sandwiched structure is quite efficient in terms of panel buckling and cylinder wrinkling, which requires require only one frame over the length of $7.1 \mathrm{~m}$ of the aft fuselage, whereas the stiffened structure requires at least five to six numbers of frames, where in terms of weight ratio per section length the stiffened structure has an advantage over the sandwiched one, which may not be very practical where a fuselage structure require a quite numbers of frames to hold floor and wings etc. The only advantage of sandwich structure over the stiffened one seems to be in terms of manufacturing. Apart from the design tradeoff study there are few technical issues, which are found in the optimization algorithm and to be addressed in future.

\section{Appendix: Panel and Section Level Constraints}

The buckling and strength constraints are being checked by the optimizer at every level i.e. from local ply level (material failure) to the skin and stiffener buckling, and further to the global buckling of a panel. In the $2^{\text {nd }}$ layer of optimization algorithm it checks the constraints of cylinder buckling due to the torsion loads.

\section{Material Failure}

Material failure is evaluated with the Tsai-Hill (TH) criterion $^{7,10}$ :

$$
\mathrm{TH}=\frac{\sigma_{x}^{2}}{X^{2}}-\frac{\sigma_{x} \sigma_{y}}{X Y}+\frac{\sigma_{y}^{2}}{Y^{2}}+\frac{\tau_{x y}^{2}}{S^{2}}
$$

where $\sigma, \tau, X, Y$, and $S$ are applied normal stress, applied normal shear stress, material maximum normal stress in $x$ direction, material maximum normal stress in $y$ direction, and material maximum shear stress, respectively.

\section{Skin Buckling}

The stability of the skin or panel facing is mainly activated for a foam-filled stiffened panel optimization problem and evaluated by assuming that the long edges of length $L_{S}$ of a long plate (skin) are built-in, where the compressive loads should not exceed the critical buckling loads $N_{x c r}{ }^{10}$ :

$$
N_{x_{c r}}=\frac{\pi^{2}}{w_{1}^{2}}\left[4.53 \sqrt{D_{(1,1)} D_{(2,2)}}+2.62\left(D_{(1,2)}+2 D_{(6,6)}\right)\right]
$$

where $D$ is the stiffness matrix of the laminate of the skin only, and $w_{1}$, as shown in Table 1 , is the stiffener spacing.

\section{Foam-Filled Stiffener/Panel Face Wrinkling}

The face wrinkling of foam filled panel or stiffener is a local phenomenon. For an isotropic core material with an isotropic facing, the critical load against the wrinkling is defined $\operatorname{as}^{10}$ : 


$$
N_{c r}=1.5 \sqrt[3]{\frac{2 D_{(1,1)} a^{2}}{\pi^{2}}} \quad \text { where } \quad a=\frac{2 \pi E_{c}}{\left(3-v_{c}\right)\left(1+v_{c}\right)}
$$

where $D, E_{c}$, and $\nu_{\mathrm{c}}$ are the stiffness matrix of the facing, Young modulus and Poisson's ratio of the core material, respectively.

Panel (Global) Buckling

Critical buckling load for a four sided simply supported panel in compression is expressed as ${ }^{10}$ :

$$
N_{x_{c r}}=\frac{\pi^{2}}{L_{s}^{2}}\left[D_{(1,1)} \frac{L_{s}^{2}}{w^{2}}+D_{(2,2)} \frac{w^{2}}{L_{s}^{2}}+2\left(D_{(1,1)}+2 D_{(6,6)}\right)\right]
$$

where $w$ is the total width of the panel. The buckling load due to shear loads is expresses as ${ }^{10}$ :

$$
\begin{gathered}
N_{x y_{c r}}=\frac{4}{L_{s}^{2}} \sqrt[4]{D_{(1,1)} D_{(2,2)}^{3}}(15.07+7.08 K) \text { when } K \leq 1 \\
N_{x y_{c r}}=\frac{4}{L_{s}^{2}} \sqrt[4]{D_{(2,2)}\left(D_{(1,2)}+2 D_{(6,6)}\right)}\left(18.59+\frac{3.56}{K}\right) \text { when } K>1 \\
K=\frac{2 D_{(6,6)}+D_{(1,2)}}{\sqrt{D_{(1,1)} D_{(2,2)}}}
\end{gathered}
$$

where

\section{Torsion Buckling}

The value of torsion load at which buckling can occur is expressed as ${ }^{3}$ :

$$
T_{c r}=4 \pi R^{2} t_{1} \tau_{c r}\left[0.8 K_{s} E_{f} \frac{\left(h+t_{1}-t_{2}\right)}{R}\right]
$$

where $E_{f}$ is the Young modulus of the facing. The buckling coefficient $K_{s}$ is a function of section length $L_{s}$ and analytical expressed in the Eq. (98) of the Ref. 15. It can also be determined by using the Fig 4.5-3 of Ref. 16.

\section{References}

${ }^{1}$ Watson, J. C., “AV-8B composite fuselage design,” J of Aircraft, Vol. 19, No. 3, March 1986, pp. 235-238.

${ }^{2}$ Jhonson, R. W., Thomson, W. L., and Wilson, R. D., "Study on utilization of advanced composites in fuselage structures of large transports", NASA CR-172406, 1985.

${ }^{3}$ van Tooren, M. J. L., Sandwich Fuselage Design, PhD Thesis, Delft University of Technology, 1998.

${ }^{4}$ Collier, C., "Stiffness, Thermal Expansion, and Thermal Bending Formulation of Stiffened, FiberReinforced Composite Panels", AIAA-93-1569-CP, Apr 19-22, 1993, pp. 2279-2290.

${ }^{5}$ Tuttle, M. E., and Zabinsky, Z. B., "Methodologies for Optimal Design of Composite Fuselage Crown Panels", AIAA-94-1493-CP, Apr 18-20, 1994, pp. 1394-1405.

${ }^{6} \mathrm{La}$ Rocca, G., and van Tooren, M. J. L., "Enabling distributed multidisciplinary design of complex products: a KBE approach" J of Design Research, Vol. 5, No. 3, 2007, pp. 1605-1613.

${ }^{7}$ Schut, E. J., and van Tooren, M. J. L., "Design "Feasilization" using knowledge-based engineering and optimization techniques", $J$ of Aircraft, Vol. 44, No. 6, November-December 2007, pp. 1776-1786.

${ }^{8}$ Baluch, H. A., Lisandrin, P., Slingerland, R., and van Tooren, M., "Effects of Flexibility on Aircraft Dynamic Loads and Structural Optimization", 45th AIAA Aerospace Sciences Meeting and Exhibit, 8-11 January 2007, Reno, USA.

${ }^{9}$ Matlab Software Package, Version 7.0 - Release 14, The Mathworks, Nathic, MA, USA, 2004.

${ }^{10}$ Kollár, L. P., and Springer, G. S., Mechanic of Composite Stuctures, Cambridge University Press, Cambridge UK, 2003, Chaps. 4, 5. 
${ }^{11}$ MSC/Nastran Superelement Users' Guide, V2005, MSC Software Corporation, CA, USA, 2005.

${ }^{12}$ Schut, E. J., "Development of FISST: An initial structural sizing tool for a fuselage", Version 1, DAR, Faculty of Aerospace Engineering, Delft University of Technology, August 2007.

${ }^{13}$ L. Meirovitch, and I. Tuzcu, "Control of Flexible Aircraft Executing Time-Dependent Maneuvers," J of Guidance, Control and Dynamics, Vol. 28, No. 6, Nov-Dec. 2005, pp. 1291-1300.

${ }^{14}$ L. Meirovitch, and I. Tuzcu, "Integrated Approach to the Dynamics and Control of Maneuvering Flexible Aircraft," NASA CR-2003-211748, 2003.

${ }^{15}$ Weingarten, V. I, Seide, P., and Peterson, J. P. "Buckling of thin-walled circular cylinders", NASA SP-8007, August 1968.

${ }^{16}$ Sullins, R. T., Smith, G. W., and Spier, E. E., "Manual for structural stability analysis of sandwich plates and shells", NASA CR-1457, 1969. 